\title{
Effect of Initial Spin Polarization on Spin Dephasing and the Electron $g$ Factor in a High-Mobility Two-Dimensional Electron System
}

\author{
D. Stich, ${ }^{1}$ J. Zhou, ${ }^{2}$ T. Korn, ${ }^{1}$ R. Schulz, ${ }^{1}$ D. Schuh, ${ }^{1}$ W. Wegscheider, ${ }^{1}$ M. W. Wu, ${ }^{2, *}$ and C. Schüller ${ }^{1, \dagger}$ \\ ${ }^{1}$ Institut für Experimentelle und Angewandte Physik, Universität Regensburg, D-93040 Regensburg, Germany \\ ${ }^{2}$ Hefei National Laboratory for Physical Sciences at Microscale and Department of Physics, \\ University of Science and Technology of China, Hefei, Anhui, 230026, China
}

(Received 19 December 2006; published 24 April 2007)

\begin{abstract}
We have investigated the spin dynamics of a high-mobility two-dimensional electron system (2DES) in a GaAs- $\mathrm{Al}_{0.3} \mathrm{Ga}_{0.7} \mathrm{As}$ single quantum well by time-resolved Faraday rotation in dependence on the initial degree of spin polarization, $P$, of the 2DES. From $P \sim 0$ to $P \sim 30 \%$, we observe an increase of the spin dephasing time, $T_{2}^{*}$, by an order of magnitude, from about $20 \mathrm{ps}$ to $200 \mathrm{ps}$, in good agreement with theoretical predictions by Weng and Wu [Phys. Rev. B 68, 075312 (2003)]. Furthermore, also the electron $g$ factor is found to decrease for increasing $P$. Fully microscopic calculations reproduce the most salient features of the experiments, i.e., a dramatic decrease of spin dephasing and a moderate decrease of the electron $g$ factor with increasing $P$. We show that both results are determined dominantly by the HartreeFock contribution of the Coulomb interaction.
\end{abstract}

DOI: 10.1103/PhysRevLett.98.176401

PACS numbers: 71.70.Ej, 39.30.+w, 73.20.-r, 85.75.-d

The spin degrees of freedom in semiconductors have largely been explored in recent years, motivated by potential applications in spintronics or quantum computational devices $[1,2]$. Very naturally, the spin dephasing and spin relaxation is of utmost interest in this field. For the paradigm spintronics device, the so-called spin transistor, as proposed by Datta and Das [3], injection and control of spin-polarized electrons into a field-effect semiconductor transistor structure is required. In a number of pioneering experiments, Kikkawa and Awschalom et al. [4,5] have demonstrated that extraordinarily long spin relaxation times can be achieved in GaAs bulk material [4] or in IIVI quantum wells [5] by using doping levels close to the metal-to-insulator transition. This provides on the one hand an outstanding means for manipulation of optically excited spins. On the other hand, however, a high impurity concentration seems to be contradictory for a transistor device, where highly mobile charge carriers are required for a minimum of dissipation processes. Definitely, another pioneering step into this direction was the experimental verification of a strongly reduced spin dephasing in GaAsbased quantum-well structures, which are grown in the [110] crystal direction by Ohno et al. [6,7]. Up to that point, the commonly used theoretical considerations, employing the D'yakonov-Perel' (DP) spin relaxation mechanism [8], predicted the absence of spin relaxation due to this mechanism for spins aligned parallel to this particular crystal direction. However, it was pointed out by $\mathrm{Wu}$ and Kuwata-Gonokami [9] that in the presence of a magnetic field in the Voigt configuration, there is still inhomogeneous broadening which can result in a finite spin dephasing contribution due to the DP mechanism. Moreover, in particular, in high-mobility two-dimensional electron systems
(2DES), as favorable components of field-effect transistorlike structures, electron-electron interaction can play a major role. This is because, as first pointed out by $\mathrm{Wu}$ and Ning [10], any scattering including the Coulomb scattering can cause an irreversible spin dephasing in the presence of inhomogeneous broadening. Recently, also for standard [001]-oriented $n$-doped quantum wells, the importance of the electron-electron scattering for spin relaxation and dephasing was demonstrated by Glazov and Ivchenko [11] from perturbation theory and Weng and $\mathrm{Wu}$ [12] from a fully microscopic many-body approach. In most of the previous theoretical and experimental studies, the spin systems are near the equilibrium, i.e., the spin polarization is very small and there is no or a small external electric field parallel to the quantum wells. Nevertheless, Weng and $\mathrm{Wu}$ investigated the spin kinetics far away from the equilibrium $[12,13]$ by setting up and numerically solving the kinetic spin Bloch equations [14] with all the scattering, especially the electron-electron Coulomb scattering explicitly included. One of the predictions of the spin dephasing far away from the equilibrium is that the spin dephasing is greatly suppressed by increasing the initial spin polarization. This effect originates from the Hartree-Fock (HF) contribution of the Coulomb interaction which serves as an effective magnetic field along the $z$ axis. This effective magnetic field is greatly increased with the spin polarization and therefore blocks the spin precession due to the lack of detuning [12].

In this Letter, we manage to realize the large spin polarization experimentally and show that the proposed effect does exist. Spin-polarized carriers are injected at the Fermi level of the 2DES via optical pumping with circularly polarized light. The ensemble spin dephasing 
time $T_{2}^{*}$ is experimentally determined via time-resolved Faraday rotation (TRFR). We find a strong increase of $T_{2}^{*}$ with increasing initial spin polarization in good agreement with the fully microscopic theory [12]. Furthermore, also the variation of the electron $g$ factor with degree of spin polarization shows, both in experiment and theory, the same tendency. This verifies and underlines the importance of electron-electron interaction for electron spin dephasing in high-mobility 2DES.

Our sample is a [001]-grown, $20 \mathrm{~nm}$-wide, one-sided modulation-doped $\mathrm{GaAs}-\mathrm{Al}_{0.3} \mathrm{Ga}_{0.7}$ As single quantum well. The electron density and mobility at $T=4.2 \mathrm{~K}$ are $n_{e}=2.1 \times 10^{11} \mathrm{~cm}^{-2}$ and $\mu_{e}=1.6 \times 10^{6} \mathrm{~cm}^{2} / \mathrm{V} \mathrm{s}$, respectively. For measurements in transmission geometry, the sample was glued onto a glass substrate with an optical adhesive, and the substrate and buffer layers were removed by selective etching. The sample was mounted in the ${ }^{3} \mathrm{He}$ insert of a superconducting split-coil magnet cryostat. For the TRFR measurements, two laser beams from a modelocked Ti:sapphire laser, which is operated at $80 \mathrm{MHz}$ repetition rate, were used. The laser pulses had a temporal length of about $600 \mathrm{fs}$ each, resulting in a spectral width of about 3-4 meV, which allowed for a resonant excitation. The laser wavelength was tuned to excite electrons from the valence band to states slightly above the Fermi energy of the host electrons in the conduction band. Both laser beams were focused to a spot of approximately $30 \mu \mathrm{m}$ diameter on the sample surface. The pump pulses were circularly polarized in order to create spin-oriented electrons in the conduction band, with spins aligned perpendicular to the quantum-well plane. Average pump powers between about $100 \mu \mathrm{W}$ and $6 \mathrm{~mW}$ were used to create different densities, $n_{\mathrm{ph}}$, of photoexcited, spin-aligned electrons. We have estimated the total densities, $n_{\mathrm{ph}}^{\text {tot }}$, of electron-hole pairs, created by a pump pulse to be between about $n_{\mathrm{ph}}^{\text {tot }}=9 \times 10^{9} \mathrm{~cm}^{-2}$ for the lowest and $n_{\mathrm{ph}}^{\text {tot }}=6 \times$ $10^{11} \mathrm{~cm}^{-2}$ for the highest pump intensities [15]. Referring to $k \cdot p$ calculations of Pfalz et al. [16], we have determined for our $20 \mathrm{~nm}$-wide GaAs well the densities of spinaligned electrons $n_{\mathrm{ph}}$ by multiplying $n_{\mathrm{ph}}^{\text {tot }}$ by a factor of 0.4 to account for heavy-light hole mixing in the valence band. The resulting degree of initial spin polarization of electrons was then calculated via the relation $P=n_{\mathrm{ph}} /\left(n_{e}+n_{\mathrm{ph}}^{\text {tot }}\right)$. The intensity of the linearly polarized probe pulses was kept constant at an average power of about $0.5 \mathrm{~mW}$, and the rotation of the probe polarization due to the Faraday effect was measured by an optical bridge.

Figure 1(a) displays measured TRFR traces at a temperature of $T=4.5 \mathrm{~K}$ for different degrees of initial electron spin polarization, $P$. As described above, $P$ was set by the average pump powers applied in the pump beam. Thus, from bottom to top spectra in Fig. 1(a), together with $P$, also the total electron densities increase (numbers are given in the figure caption) [17]. In particular, at higher pump intensities, a biexponential decay can be found. There is a very fast decay of the TRFR signal within the first few

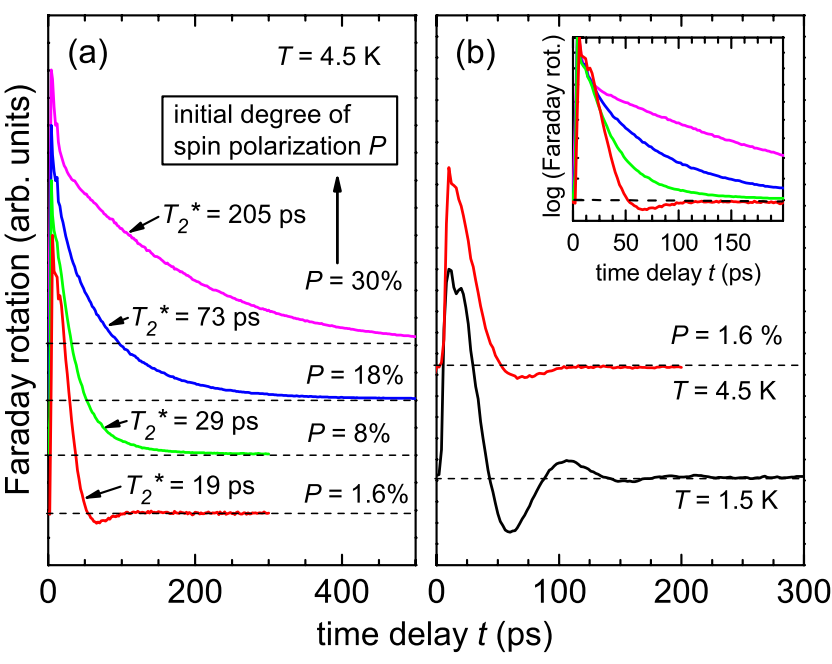

FIG. 1 (color online). (a) Normalized TRFR traces for different degrees of initial electron spin polarization, $P$. The total densities of electrons, $n_{\text {tot }}=n_{e}+n_{\mathrm{ph}}^{\text {tot }}$, are 2.19, 2.66, 3.83, 8.39 (in units of $10^{11} \mathrm{~cm}^{-2}$ ) for $P=1.6 \%, 8 \%, 18 \%, 30 \%$, respectively. (b) Comparison of two traces with $P=1.6 \%$ at two different temperatures. For the lowest temperature, a coherent electron spin oscillation due to the Dresselhaus-Rashba spinorbit field is clearly seen. The inset shows a semilogarithmic plot of the data displayed in (a). Note that at low $P$ the zero-field oscillation is superimposed to the decay.

picoseconds, followed by a second, much longer decay. We attribute the fast initial decay to the spin relaxation of the photoexcited holes, which lose their initial spin orientation extremely fast. The second long decay is due to electron spin relaxation. The extracted electron spin dephasing times $T_{2}^{*}$, given in Fig. 1(a), increase significantly with increasing $P$. For the lowest $P$ in Fig. 1(a), a very weak oscillation can be observed. As Fig. 1(b) shows, this oscillation increases, when the temperature is lowered from $4.5 \mathrm{~K}$ to $1.5 \mathrm{~K}$. A very similar zero-field spin oscillation was previously reported by Brand et al. [18]. The oscillatory signal is due to a coherent oscillation of the excited electron spins about an effective spin-orbit field caused by $k$-linear terms in the Rashba-Dresselhaus Hamiltonian. The inset of Fig. 1(b) shows a semilogarithmic plot of the experimental curves, displayed in Fig. 1(a). Note that, in particular, at low $P$ the zero-field oscillation is superimposed on the decay. At high $P$, the exponential decay is clearly visible.

In order to understand the experimental results, we have performed a fully microscopic calculation on the corresponding cases by setting up and numerically solving the kinetic spin Bloch equations $[12,13,19]$. In the calculation, we only consider the DP mechanism by including the Dresselhaus term [20]. All the spin-conserving scattering such as the electron-acoustic-phonon, electron-nonmagnetic-impurity, electron-electron and electron-heavy-hole Coulomb scatterings as well as spin-flip electron-hole Coulomb scattering [the so-called Bir-Aronov-Pikus (BAP) mechanism [21]] are included. The impurity den- 
sity is determined from the Hall mobility measured in the experiment. The details of the calculation can be found in Ref. [19], except now the screening is expanded to include the contribution from heavy holes. It is seen that the theoretical calculations, displayed in Fig. 2(a), qualitatively reproduce the experimental findings very well: the very weak zero-field spin oscillation is observed for small $P$ and disappears for higher $P$. Furthermore, the dramatic decrease of the spin dephasing with $P$, first predicted by Weng and Wu in Ref. [12], is reproduced in the calculation. This quick decrease of the spin dephasing with the spin polarization was suggested to be due to the HF contribution from the Coulomb interaction [12]. This can be seen clearly by comparing the corresponding curves in Fig. 2(a) with Fig. 2(b) where the HF term is removed.

It is seen that a fitting parameter $\tau$ is introduced in Fig. 2 so that we may obtain the same $T_{2}^{*}$ as the experiment. This phenomenological parameter is used to represent (i) additional sources of spin dephasing, not included in the present model, such as the effect from the asymmetric quantum well in the experiment (the Rashba term), the multisubband effect at large $P$ and (ii) the fact that with the finite laser pulse width of about 3-4 meV in the experiment, we cannot probe the complete energy range above the Fermi energy, as we do in the calculations. It is seen that $\tau$ increases with increasing $P$ : for high polarizations the photoexcited hole density becomes much higher and

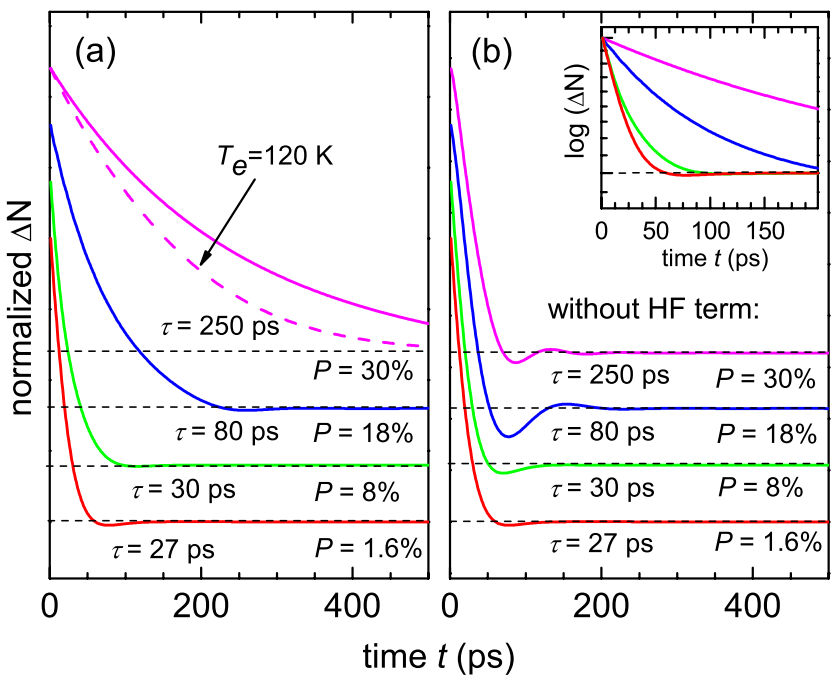

FIG. 2 (color online). (a) Calculated spin decay curves for the experimental parameters, like initial spin polarization, total electron densities, electron mobility, and temperature $T=$ 4.5 K (solid lines). In the calculation, a phenomenological decay time is incorporated as a single fitting parameter $\tau$ (see text). The dashed curve for $P=30 \%$ is calculated for a hot electron temperature of $T_{e}=120 \mathrm{~K}$. (b) Same as (a) but calculated without the HF term. In particular, for large $P$ the decay is much faster than in the experiments [cf. Fig. 1(a)]. The inset shows the data, displayed in (a), in a semilogarithmic plot. At low $P$ the zero-field oscillations are superimposed. consequently the BAP mechanism becomes stronger. Therefore, the remaining unaccounted mechanisms become less significant, and hence $\tau$ becomes larger. It is noted that in Fig. 2 the same parameters $\tau$ are used for both calculations with and without the HF term. Moreover, as the actual temperature in the laser spot can be higher than what we measure at the sample holder $(4.5 \mathrm{~K})$, for the highest pump power $P=30 \%$, we present another calculation by taking hot electron temperature $T_{e}=120 \mathrm{~K}$ as a dashed curve [22]. It shows that, theoretically, the spin dephasing time decreases with temperature, which is in opposite to the temperature dependence at small spin polarization [19]. This indicates that the enhancement of the spin dephasing time with the spin polarization is solely due to the HF term even if the temperature is raised at high $P$. Finally, as a summary, in Fig. 3 the spin dephasing times, as extracted from the curves in Figs. 1 and 2 are displayed versus $P$ and total electron density $n_{\text {tot }}$. As mentioned above, a phenomenological $\tau$ (see Fig. 2) is included in the calculations to derive the same dephasing times as in the experiment. We want to emphasize that the important result here is the striking difference of the calculations with (solid dots) and without the HF term (open circles) for the same $\tau$ [23].

We now turn to the discussion of experiments in an external magnetic field, applied in Voigt geometry, i.e., parallel to the quantum-well plane. Figure 4(a) shows TRFR traces for fixed magnetic field of $B=4 \mathrm{~T}$ but different spin polarizations $P$. One can clearly see that, as $P$ is increased, the precession period increases [see arrow in Fig. 4(a)]. We plot in Fig. 4(b) the polarization dependence of the electron $g$ factor which we have extracted from the experimental curves. One can see that $g$ decreases with $P$

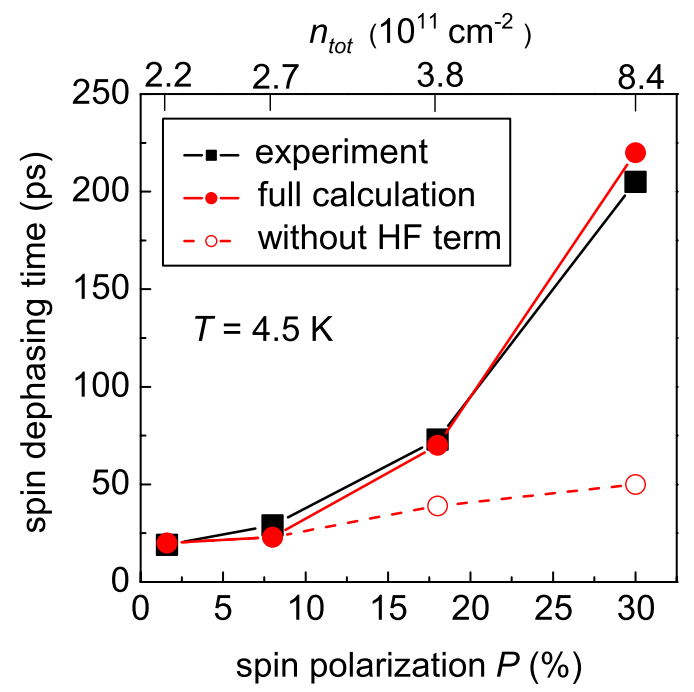

FIG. 3 (color online). Comparison of spin dephasing times, as extracted from the experimental curves in Fig. 1(a) (ם), and from the calculated curves in Fig. 2(a) with (O) and Fig. 2(b) without $(\bigcirc)$ the $\mathrm{HF}$ term. In both calculations, phenomenological decay times, $\tau$, as indicated in Fig. 2, were included. 

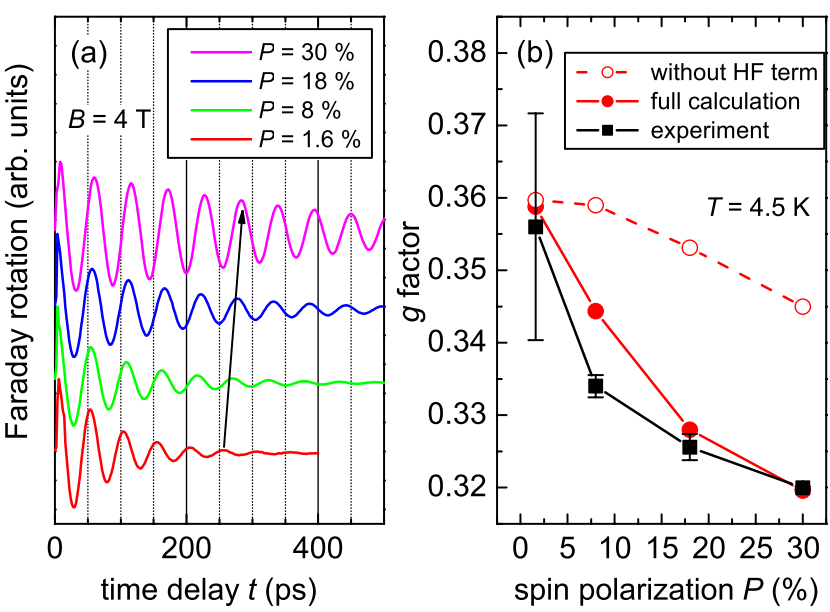

FIG. 4 (color online). (a) TRFR measurements at $B=4 \mathrm{~T}$ for different $P$. An increase of the electron precession period with increasing $P$ is clearly observed (arrow). (b) Comparison of electron $g$ factors for different polarization degrees $P$, as extracted from the experiments $(\boldsymbol{\square})$ and the calculations with $(\mathbf{O})$ and without $(\bigcirc) \mathrm{HF}$ term.

by nearly $10 \%$ while $P$ is increasing from $1.6 \%$ to $30 \%$. The calculations with and without the HF term also indicate the marked variation of $g$, with the variation for the former case being larger than the latter one. This shows that in the weak scattering limit, both the effective magnetic fields from the Dresselhaus term and the HF term change the frequency of Larmor precession.

In conclusion, we have investigated the spin dephasing and the electron $g$ factor of a high-mobility 2DES in dependence of an initial electron spin polarization (especially far away from the equilibrium). Experimentally, the initial spin polarization was realized by optically pumping spin-aligned electrons into states above the Fermi energy of the 2DES. We have found a marked increase of the spin dephasing time with spin polarization and a slight decrease of the electron $g$ factor. The experimental results could be confirmed by fully microscopic calculations, employing the kinetic spin Bloch equations. Importantly, it turned out that in the calculations the HF contribution from the Coulomb interaction plays a dominant role and is responsible for the dramatic increase of the spin dephasing time at large spin polarization. It can by no means be neglected in order to correctly account for the experimental observations.

We gratefully thank Jaroslav Fabian and R. T. Harley for valuable discussions. This work was supported by the Deutsche Forschungsgemeinschaft via GrK 638, Grants No. Schu1171/1-3, No. SFB 689, and No. SPP1285. M.W.W. was supported by the Natural Science Foundation of China under Grant No. 10574120, the National Basic Research Program of China under Grant No. 2006CB922005, the Knowledge Innovation Project of Chinese Academy of Sciences, and SRFDP.
*Electronic address: mwwu@ustc.edu.cn.

${ }^{\dagger}$ Electronic address: christian.schueller@physik.uniregensburg.de.

[1] Semiconductor Spintronics and Quantum Computation, edited by D. D. Awschalom, D. Loss, and N. Samarth, Nanoscience and Technology (Springer, New York, 2002), and references therein.

[2] I. Zutic, J. Fabian, and S. Das Sarma, Rev. Mod. Phys. 76, 323 (2004), and references therein.

[3] S. Datta and B. Das, Appl. Phys. Lett. 56, 665 (1990).

[4] J. M. Kikkawa and D. D. Awschalom, Phys. Rev. Lett. 80, 4313 (1998).

[5] J. M. Kikkawa, I. P. Smorchkova, N. Samarth, and D. D. Awschalom, Science 277, 1284 (1997).

[6] Y. Ohno, R. Terauchi, T. Adachi, F. Matsukura, and H. Ohno, Phys. Rev. Lett. 83, 4196 (1999).

[7] see also, S. Döhrmann, D. Hägele, J. Rudolph, M. Bichler, D. Schuh, and M. Oestreich, Phys. Rev. Lett. 93, 147405 (2004).

[8] M. I. D'yakonov and V. I. Perel', Zh. Eksp. Teor. Fiz. 60, 1954 (1971) [Sov. Phys. JETP 33, 1053 (1971)].

[9] M.W. Wu and M. Kuwata-Gonokami, Solid State Commun. 121, 509 (2002).

[10] M. W. Wu and C. Z. Ning, Eur. Phys. J. B 18, 373 (2000).

[11] M. M. Glazov and E. L. Ivchenko, JETP Lett. 75, 403 (2002).

[12] M.Q. Weng and M.W. Wu, Phys. Rev. B 68, 075312 (2003).

[13] M.Q. Weng, M. W. Wu, and L. Jiang, Phys. Rev. B 69, 245320 (2004).

[14] M. W. Wu and H. Metiu, Phys. Rev. B 61, 2945 (2000).

[15] For estimation of the densities of photoexcited electronhole pairs, $n_{\mathrm{tot}}^{\mathrm{ph}}$, the energy-dependent absorption coefficient of the sample, and the laser spot size were measured. From this, and the laser beam intensity, $n_{\text {tot }}^{\mathrm{ph}}$ was calculated.

[16] S. Pfalz, R. Winkler, T. Nowitzki, D. Reuter, A. D. Wieck, D. Hägele, and M. Oestreich, Phys. Rev. B 71, 165305 (2005).

[17] The measured photocarrier lifetime (not shown here) was on the order of 1 ns so that we assume that lifetime effects on the spin relaxation are of minor importance.

[18] M. A. Brand, A. Malinowski, O.Z. Karimov, P. A. Marsden, R. T. Harley, A. J. Shields, D. Sanvitto, D. A. Ritchie, and M. Y. Simmons, Phys. Rev. Lett. 89, 236601 (2002).

[19] J. Zhou, J.L. Cheng, and M. W. Wu, Phys. Rev. B 75, 045305 (2007).

[20] G. Dresselhaus, Phys. Rev. 100, 580 (1955).

[21] G. L. Bir, A. G. Aronov, and G. E. Pikus, Zh. Eksp. Teor. Fiz. 69, 1382 (1975) [Sov. Phys. JETP 42, 705 (1975)].

[22] We have estimated the increase of electron temperature with laser pump power by analyzing the photoluminescence line shape at similar excitation conditions. From this we get a moderate increase from about $T_{e}=7 \mathrm{~K}$ to $T_{e}=$ 18 K for $P=1.6 \%$ to $P=30 \%$.

[23] Moreover, without $\tau$, the curve for the results without the HF term in Fig. 3 (open circles) appears even flatter than displayed, which underlines the importance of this term. 